\title{
Number of representations related to a linear recurrent basis
}

\author{
by
}

\section{Jean Marie Dumont $\dagger$, Nikita Sidorov (St. Petersburg) and Alain Thomas (Marseille)}

0. General results and notations. We first present a general statement on the number of representations related to a linear recurrent basis. Let $r$ be an integer, $r \geq 1$, and let $a_{1}, \ldots, a_{r}$ be reals. We consider a sequence $\left(G_{k}\right)_{k \geq 0}$ such that for any $k \geq r$,

$$
G_{k}=a_{1} G_{k-1}+\ldots+a_{r} G_{k-r} .
$$

Let $P(X)=X^{r}-a_{1} X^{r-1}-\ldots-a_{r}$; we will assume the following hypothesis to be satisfied: there is only one root of $P(X)$ with the maximum modulus; it is a real $\phi>1$ and has multiplicity 1 . Thus, the limit

$$
\gamma=\lim _{k \rightarrow \infty}\left(G_{k} \phi^{-k}\right)
$$

exists, and we assume it to be positive.

Now let $q$ be an integer, $q \geq 2$, and consider the alphabet $\mathcal{A}_{q}=\{0,1, \ldots$ $\ldots, q-1\}$. We denote an infinite word on $\mathcal{A}_{q}$ by $\varepsilon_{0} \varepsilon_{1} \ldots$ We define $f(x)$, the number of $q$-representations of a real $x$ in the basis $\left(G_{k}\right)$, as

$$
f(x)=\#\left\{\varepsilon_{0} \varepsilon_{1} \ldots: \sum_{i=0}^{\infty} \varepsilon_{i} G_{i}=x\right\} .
$$

Since $G_{k} \sim \gamma \phi^{k}$ with $\gamma>0$ and $\phi>1$, these sequences are such that $\varepsilon_{i} \equiv 0$ for $i$ large enough. For any $x \in \mathbb{R}$, the set of $x^{\prime}<x$ such that $f\left(x^{\prime}\right) \neq 0$ is finite, and we can define the summation function

$$
\Phi(x)=\sum_{x^{\prime}<x} f\left(x^{\prime}\right) .
$$

1991 Mathematics Subject Classification: Primary 11A67; Secondary 11B75, 60E05, $60 \mathrm{E} 10$.

$\dagger$ Prof. Dumont passed away on September 18, 1997.

The second author was supported in part by the French foundation Pro Mathematica. 
We will prove in Section 2 the existence of a unique periodic function $H$ with period 1 such that

$$
\Phi(x)=x^{\log _{\phi} q} H\left(\log _{\phi} x\right)+o\left(x^{\log _{\phi} q}\right), \quad x \rightarrow \infty .
$$

The function $H$ is continuous, a.e. differentiable and $\Phi(x) \asymp x^{\log _{\phi} q}$ as $x \rightarrow$ $\infty$, or, equivalently, $H(x)>0$ for any $x \in \mathbb{R}$.

In Section 3 we give an upper bound for the remainder term, depending on the constant $\delta$ such that $f(x)=O\left(x^{\delta}\right)$, and in Section 4 a lower bound for the Lipschitz exponent of $H$ will be obtained under the same hypothesis.

Section 5 concerns properties of $H$ for some cases of $\phi$. If $\phi$ is an irrational Pisot number, then $H$ is not differentiable on a certain continuum of points. For $\phi \in \mathbb{N}$ and $G_{k}=\phi^{k}$ with $q$ not divisible by $\phi$ we have the same result. On the contrary, in the case when $\phi$ divides $q$, the function $H$ is absolutely continuous.

In Section 6 we study the general case of an integer base of the form $G_{k}=d^{k}$, i.e. a radix base. The formula

$$
\Phi(x)=x^{\alpha} H\left(\log _{d} x\right)+O\left(x^{\beta}\right)
$$

holds with $\alpha=\log _{d} q$ and $\beta=\log _{d}[(q-1+d) / d]$, and we show that in a general situation the order of the remainder term $R(x):=\Phi(x)-x^{\alpha} H\left(\log _{d} x\right)$ cannot be improved. In the case of a radix base we completely reduce the problems of finding the best possible exponent of the remainder term and the minimal Lipschitz exponent for the function $H$ to estimating $f(n)$. More precisely, it is proved that

(1) $\delta_{f}:=\inf \left\{\delta: R(x)=O\left(x^{\delta}\right)\right\}=\inf \left\{\delta: f(n)=O\left(n^{\delta}\right)\right\}$,

(2) the Lipschitz exponent for $H$ equals $\alpha-\delta_{f}$ if $\delta_{f}>\alpha-1$.

Thus, it proves to be sufficient to estimate $f(n)$. We present the best possible estimates in the following cases:

(i) $d$ divides $q$,

(ii) $d=2, q=5$,

(iii) $q \leq d^{2}$.

For instance, in the simple case $d=2, q=3$ we prove that the exact order of the remainder term is $x^{\beta^{\prime}}$ with $\beta^{\prime}=\log _{2} \frac{\sqrt{5}+1}{2}$.

Note that in the case $q=d^{m}$ for some $m \geq 1$, we give an explicit asymptotic formula for $f(n)$.

Besides, we associate with any radix base with $q>d$ a finite set of 0-1 matrices $M_{0}, M_{1}, \ldots, M_{d-1}$ so that the growth of $f(n)$ can be represented by means of the growth of the quantity $\left\|M_{\varepsilon_{0}} M_{\varepsilon_{1}} \ldots M_{\varepsilon_{k}}\right\|$, where $n=\sum_{i=0}^{k} \varepsilon_{i} d^{i}$ is the ordinary $d$-ary expansion of a positive integer $n$. Note that certain problems closely connected with the study of Mahler-type sequences were considered in the recent work $[\mathrm{DF}]$. 
The case of the Fibonacci sequence, i.e. $G_{k}=F_{k}$ defined by $F_{0}=1$, $F_{1}=2$ and $F_{k}=F_{k-1}+F_{k-2}$, was under consideration in [S]. With the alphabet $\mathcal{A}_{2}=\{0,1\}, f(n)$ is the number of representations of $n$ as a sum of distinct Fibonacci numbers for any $n \in \mathbb{N}$. Here the formula for the summation function is

$$
\Phi(x)=x^{\Lambda} H\left(\log _{\lambda} x\right)+O\left(x^{1 / 2}\right)
$$

with $\lambda=(1+\sqrt{5}) / 2 \simeq 1.62$ and $\Lambda=\log _{\lambda} 2 \simeq 1.44$. The remainder term $O\left(x^{1 / 2}\right)$ cannot be replaced by $o\left(x^{1 / 2}\right)$; the function $H$ belongs to the Lipschitz class with exponent $\Lambda-1 / 2 \simeq 0.94$. Note that the precise maximal order of the function $f$ itself is $f(x)=O\left(x^{1 / 2}\right)$ (see $[\mathrm{Pu}]$ ).

Another example of such type is $G_{k}=\lambda^{k}$ : the above formula holds with the same function $H$.

We would also like to mention that if one studies the number $g(n)$ of representations of a positive integer $n$ in a $d$-radix base with the set of all nonnegative integer digits, then the old result of N. G. Bruijn $[\mathrm{Br}]$ states that the asymptotic order of $\log g(n)$ is $\log ^{2} n$, i.e. $g(n)$ grows faster than any polynomial. The authors express their gratitude to P. Flajolet for indicating this result.

Graphic interpretation. We associate a graded graph with any sequence $\left(G_{k}\right)$ of the type described above and any $q \geq 2$ in the following way. Let the zero level of the graph contain a single vertex. Next, the $n$th level with $n \geq 1$ consists of the numbers of the form $\left\{\sum_{i=0}^{n} \varepsilon_{i} G_{i}: 0 \leq \varepsilon_{i} \leq q-1\right\}$. From any vertex exactly $q$ edges descend, and each edge is parametrized by one of the digits $0, \ldots, q-1$ (normally the leftmost one corresponds to 0 ). Thus, any finite path $\left(\varepsilon_{n}, \varepsilon_{n-1}, \ldots, \varepsilon_{0}\right)$ in this graph corresponds to one of the representations described above. We call this graph the representation graph associated with the sequence $\left(G_{n}\right)$ and the positive integer $q \geq 2$. Note that in a meaningful situation (i.e. when the function $f$ assumes values greater than 1), the graph is not a tree. For the Fibonacci case the combinatorial and ergodic properties of the representation graph are studied in detail in [AlZ] and [SV]. The representation graph for the radix case with $d=2$, $q=3$ is depicted in the last section (see Figure 1). Note that this last graph appeared for the first time in the master thesis [Bo] in connection with the study of the celebrated de Rham curve introduced in [Rh].

1. Some preliminary lemmas about representations in the basis $\left(G_{k}\right)$. We put

$$
Y\left(\varepsilon_{0}, \varepsilon_{1}, \ldots\right):=\sum_{i=0}^{\infty} \varepsilon_{i} \phi^{-i}
$$

where $\varepsilon_{i}$ assumes the integer values between 0 and $q-1$ with probability 
$1 / q$ each. Let $F_{Y}$ be the distribution function of the random variable $Y$; its continuity and purity is a consequence of the theorem stating that a series of discrete stochastically nonconstant random variables is continuous and either absolutely continuous or singular (the "Law of Pure Types", see [JW, Theorem 35]).

We first give, in Lemma 1.1 below, some estimates for $G_{k}$ and $\sum_{i=0}^{k} \varepsilon_{i} G_{i}$. Let the roots of $P(X)$ be denoted as $\phi_{1}=\phi, \phi_{2}, \ldots, \phi_{d^{\prime}}$, and let $\alpha_{i}$ be the order of multiplicity of $\phi_{i}$. We suppose

$$
\begin{aligned}
& \phi>\left|\phi_{2}\right| \geq\left|\phi_{i}\right| \quad \forall i \in\left\{3, \ldots, d^{\prime}\right\}, \\
& \alpha_{2} \geq \alpha_{i} \quad \forall i \in\left\{3, \ldots, d^{\prime}\right\} \quad \text { such that }\left|\phi_{2}\right|=\left|\phi_{i}\right|, \\
& \gamma=\lim G_{k} \phi^{-k}>0 .
\end{aligned}
$$

We write $g(x) \asymp h(x)$ if $g(x)=O(h(x))$ and $h(x)=O(g(x))$. We set

$$
\varrho=\max \left(\left|\phi_{2}\right|, 1\right), \quad \alpha^{\prime}= \begin{cases}\alpha_{2}-1, & \left|\phi_{2}\right|>1, \\ \alpha_{2}, & \left|\phi_{2}\right|=1, \\ 0, & \left|\phi_{2}\right|<1\end{cases}
$$

( $\varrho=1$ and $\alpha^{\prime}=0$ if $P(X)$ has degree one),

$$
\varphi(k)=\left|\phi_{2}\right|^{k} k^{\alpha_{2}-1}, \quad \psi(k)=\varrho^{k} k^{\alpha^{\prime}}
$$

$\left(\psi(k)\right.$ is defined in such a way that $\left.\psi(k) \asymp \sum_{i=0}^{k} \varphi(i)\right)$.

Lemma 1.1. There exist two positive constants $K_{1}$ and $K$ such that

(i) $\left|G_{k}-\gamma \phi^{k}\right| \leq K_{1} \varphi(k)$,

(ii) for any $k \in \mathbb{N}$ and an infinite word $\varepsilon_{0} \varepsilon_{1} \ldots$ on the alphabet $\mathcal{A}_{q}$,

$$
\left|\sum_{i=0}^{k} \varepsilon_{i} G_{k-i}-\sum_{i=0}^{\infty} \varepsilon_{i} \gamma \phi^{k-i}\right| \leq K \psi(k) .
$$

Proof. (i) is a direct consequence of the root expression of $G_{k}$, i.e.

$$
G_{k}=\sum_{i} \sum_{j<\alpha_{i}} \lambda_{i, j} \phi_{i}^{k} k^{j}
$$

and of the general assumptions.

(ii) We have

$$
\sum_{i=0}^{k} \varepsilon_{i}\left(G_{k-i}-\gamma \phi^{k-i}\right)=O\left(\sum_{i=0}^{k} \varphi(k-i)\right) \text { and } \sum_{i=k+1}^{\infty} \phi^{k-i}=O(1),
$$

hence both expressions are $O(\psi(k))$.

The following lemma gives a relation between the functions $\Phi$ and $F_{Y}$. 
Lemma 1.2. Let $x \in \mathbb{R}$ and $k \in \mathbb{N}$ be such that $x<\gamma \phi^{k}$, let $x_{k}=$ $x-K \psi(k)$ and $x_{k}^{\prime}=x+K \psi(k)$. For $k$ large enough we have

$$
\begin{gathered}
q^{k+1} F_{Y}\left(\frac{x_{k}}{\gamma \phi^{k}}\right) \leq \Phi(x) \leq q^{k+1} F_{Y}\left(\frac{x_{k}^{\prime}}{\gamma \phi^{k}}\right), \\
\Phi\left(x_{k}\right) \leq q^{k+1} F_{Y}\left(\frac{x}{\gamma \phi^{k}}\right) \leq \Phi\left(x_{k}^{\prime}\right) .
\end{gathered}
$$

Proof. (i) Let $\varepsilon_{0} \varepsilon_{1} \ldots, x$ and $k$ be such that $\sum_{i=0}^{\infty} \varepsilon_{i} G_{i}<x<\gamma \phi^{k}$, and $j$ be such that $\varepsilon_{j} \neq 0$. Since $G_{i} \sim \gamma \phi^{i}$, the $G_{i}$ are positive for $i>i_{1}$ and we deduce from the hypothesis that

$$
\gamma \phi^{k}>G_{j}-\sum_{i=0}^{i_{1}} q\left|G_{i}\right|>\gamma \phi^{j-1}
$$

where the last inequality is valid for $j$ large enough. In other words, if $k$ is large enough, then $\varepsilon_{j}=0$ for any $j>k$, whence

$$
\begin{aligned}
\Phi(x) & =\#\left\{\varepsilon_{0} \varepsilon_{1} \ldots \varepsilon_{k}: \sum_{i=0}^{k} \varepsilon_{i} G_{i}<x\right\} \\
& =q^{k+1} m_{q}\left\{\left(\varepsilon_{0}, \varepsilon_{1}, \ldots\right): \sum_{i=0}^{k} \varepsilon_{i} G_{k-i}<x\right\},
\end{aligned}
$$

where $m_{q}$ is the uniform product measure on the compactum $\prod_{0}^{\infty}\{0, \ldots$ $\ldots, q-1\}$.

On the other hand, we have, with $y=x_{k}$ or $x_{k}^{\prime}$,

$$
F_{Y}\left(\frac{y}{\gamma \phi^{k}}\right)=m_{q}\left\{\left(\varepsilon_{0}, \varepsilon_{1}, \ldots\right): \sum_{i=0}^{\infty} \varepsilon_{i} \phi^{-i}<\frac{y}{\gamma \phi^{k}}\right\},
$$

whence (i) follows by Lemma 1.1(ii).

(ii) We can replace $x$ by $x_{k}$, and hence $x_{k}^{\prime}$ by $x$, in the second inequality in (i). Indeed, $x_{k}$ also satisfies the condition $x_{k}<\gamma \phi^{k}$. Thus, we obtain the first inequality in (ii).

We obtain the second one noting that

$$
\Phi\left(x_{k}^{\prime}\right) \geq \#\left\{\varepsilon_{0} \varepsilon_{1} \ldots \varepsilon_{k}: \sum_{i=0}^{k} \varepsilon_{i} G_{i}<x_{k}^{\prime}\right\}
$$

and using the same method as for (i).

\section{Main result}

THEOREM 2.1. There exists a unique periodic function $H$ with period 1 , well defined on the whole real line, such that 


$$
\Phi(x)=x^{\log _{\phi} q} H\left(\log _{\phi} x\right)+o\left(x^{\log _{\phi} q}\right), \quad x \rightarrow \infty .
$$

This function is positive, continuous and a.e. differentiable.

Proof. With any $x$ we associate the integer $k=k(x)$ such that $\gamma \phi^{k-1} \leq$ $x<\gamma \phi^{k}:$ it is

$$
k(x)=\left[\log _{\phi} \frac{x}{\gamma}\right]+1 .
$$

We apply Lemma 1.2(i) and note that $x /\left(\gamma \phi^{k}\right)-x_{k} /\left(\gamma \phi^{k}\right)$ and $x_{k}^{\prime} /\left(\gamma \phi^{k}\right)-$ $x /\left(\gamma \phi^{k}\right)$ tend to 0 as $x \rightarrow \infty$ and $k=k(x)$. By the uniform continuity of the distribution function, $F_{Y}(y+t)-F_{Y}(y)$ tends to 0 uniformly in $y$ as $t \rightarrow 0$. So, we deduce from Lemma 1.2(i) that

$$
\Phi(x)=q^{k(x)+1} F_{Y}\left(\frac{x}{\gamma \phi^{k(x)}}\right)+o\left(q^{k(x)+1}\right) .
$$

Thus, we obtain the desired formula with

$$
H\left(\log _{\phi} x\right)=q^{k(x)+1-\log _{\phi} x} F_{Y}\left(\frac{x}{\gamma \phi^{k(x)}}\right) .
$$

Periodicity of $H$. Clearly $H\left(1+\log _{\phi} x\right)=H\left(\log _{\phi}(\phi x)\right)=H\left(\log _{\phi} x\right)$.

Uniqueness of $H$. The above formula and the periodicity of $H$ imply

$$
H\left(\log _{\phi} x\right)=\lim _{k \rightarrow \infty} H\left(\log _{\phi}\left(x \phi^{k}\right)\right)=\lim _{k \rightarrow \infty} \Phi\left(x \phi^{k}\right)\left(x \phi^{k}\right)^{-\log _{\phi} q} .
$$

Positivity. We deduce from the definition of $H$ that

$$
H\left(\log _{\phi} x\right) \geq q^{1-\log _{\phi} \gamma} F_{Y}(1 / \phi) .
$$

The number $F_{Y}(1 / \phi)$ is positive, as there exists $k_{0}$ such that $Y\left(\varepsilon_{0}, \varepsilon_{1}, \ldots\right)<$ $1 / \phi$ if $\varepsilon_{0}=\varepsilon_{1}=\ldots=\varepsilon_{k_{0}}=0$.

Continuity. The function $H$ could be discontinuous only at $x$ such that $\log _{\phi}(x / \gamma)$ is an integer; nevertheless, if we replace in the definitions of $H$ and $k(x)$ the condition $\gamma \phi^{k-1} \leq x<\gamma \phi^{k}$ by $\gamma \phi^{k-1} / \sqrt{\phi} \leq x<\gamma \phi^{k} / \sqrt{\phi}$ (for instance), the formula of Theorem 2.1 will remain valid. Since $\log _{\phi}(x / \gamma)$ and $\log _{\phi}(x \sqrt{\phi} / \gamma)$ cannot be both integers, the (unique) function $H$ is everywhere continuous.

Differentiability. The continuity of the distribution function $F$ implies that it is a.e. differentiable, thence $H$ is a.e. differentiable. The proof is complete.

3. Improvement of the remainder term. Note first that since $f(n) \leq$ $\Phi(n+1)$, we have $f(n)=O\left(n^{\log _{\phi} q}\right)$. So, the sequence $f(n)$ cannot grow more rapidly than a polynomial. Henceforward we assume that a constant $\delta>0$ is such that 
(I) $f(x)=O\left(x^{\delta}\right)$ for $x \in \mathbb{R}_{+}$, and

(II) $\#\left\{x^{\prime}: x^{\prime} \in\left[x, x+1\left[, f\left(x^{\prime}\right) \neq 0\right\}\right.\right.$ is uniformly bounded for $x \in$ $\mathbb{R}_{+}\left({ }^{1}\right)$.

For this situation the main formula of the previous section can be improved.

Theorem 3.1. Let $\varrho$ and $\alpha^{\prime}$ be defined as in Section 1. We have

$$
\Phi(x)=x^{\alpha} H\left(\log _{\phi} x\right)+O\left(x^{\delta^{\prime}}(\log x)^{\alpha^{\prime}}\right)
$$

with $\alpha=\log _{\phi} q$ and $\delta^{\prime}=\delta+\log _{\phi} \varrho$.

Proof. We use Lemma 1.2(ii) with $k=k(x)=\left[\log _{\phi}(x / \gamma)\right]+1$. By hypothesis (II), the number of reals $y \in\left[x_{k}, x_{k}^{\prime}\right]$ such that $f(y) \neq 0$ is $O\left(x_{k}^{\prime}-x_{k}\right)$; and by hypothesis (I) we have in this case $f(y)=O\left(\left(x_{k}^{\prime}\right)^{\delta}\right)=$ $O\left(x^{\delta}\right)$. We deduce that both $\Phi(x)-\Phi\left(x_{k}\right)$ and $\Phi\left(x_{k}^{\prime}\right)-\Phi(x)$ are $O\left(x^{\delta} \psi(k)\right)$, whence

$$
q^{k(x)+1} F_{Y}\left(\frac{x}{\gamma \phi^{k(x)}}\right)=\Phi(x)+O\left(x^{\delta} \psi(k(x))\right) .
$$

It remains to note that $\psi(k(x))=\varrho^{k(x)}(k(x))^{\alpha^{\prime}}=O\left(x^{\log _{\phi} \varrho}(\log x)^{\alpha^{\prime}}\right)$.

Theorem 3.1 improves Theorem 2.1 iff $\alpha>\delta^{\prime}$. We will give a suitable estimate of the order of $f(n)$ in a certain natural case.

Proposition 3.2. Let $f(x)$ be the number of q-representations of $x$ in a certain nonnegative integer base $\left(G_{k}\right)$, with $q=[\phi]+1$. If $\phi>(1+\sqrt{5}) / 2$ and $\phi \neq 2$, then $f(n)=O(n)$.

Proof. By Lemma 1.1, there exists a constant $K^{\prime}$ such that

$$
\begin{aligned}
G_{k}-[\phi] \sum_{i=0}^{k-2} G_{i} & \geq \gamma \phi^{k}-[\phi] \gamma \sum_{i=0}^{k-2} \phi^{i}-K^{\prime} \psi(k) \\
& >\gamma \phi^{k}-[\phi] \gamma \frac{\phi^{k-1}}{\phi-1}-K^{\prime} \psi(k) \\
& >\gamma \phi^{k-1} \frac{\phi^{2}-\phi-[\phi]}{\phi-1}-K^{\prime} \psi(k) .
\end{aligned}
$$

The assumptions on $\phi$ imply that $\left(\phi^{2}-\phi-[\phi]\right) /(\phi-1)>0$, whence there is a constant $k_{1}$ such that

$$
G_{k}>[\phi] \sum_{i=0}^{k-2} G_{i}, \quad G_{k-1}>0 \text { and } G_{k} \text { is nondecreasing }
$$

for $k \geq k_{1}$.

$\left({ }^{1}\right)$ The second condition is satisfied, for instance, if $G_{k} \in \mathbb{N}$, or if $G_{k}=\phi^{k}$ with $\phi$ being a Pisot number (see $[\mathrm{Bu}])$. 
Choosing a constant $C$ satisfying the relation $f(n) \leq C n$ for any $n<$ $G_{k_{1}}$, we prove by induction the validity of this relation for $n \in \mathbb{N}$. Namely, suppose $f\left(n^{\prime}\right) \leq C n^{\prime}$ for any $n^{\prime} \leq n-1$ and let $k$ be such that $G_{k} \leq n<$ $G_{k+1}$. There exists also an integer $\varepsilon \geq 0$ such that

$$
\varepsilon G_{k-1}+[\phi] \sum_{i=0}^{k-2} G_{i}<n \leq(\varepsilon+1) G_{k-1}+[\phi] \sum_{i=0}^{k-2} G_{i} .
$$

As $n<G_{k+1}$, the representations of $n$ in the alphabet $\{0,1, \ldots,[\phi]\}$ satisfy $\varepsilon_{i}=0$ for $i \geq k+1$. If $\varepsilon_{k}=0$, we have $\varepsilon_{k-1} \geq \varepsilon+1$. With each representation of $n$ satisfying $\varepsilon_{k} \neq 0$, we associate a representation of $n-G_{k}$, replacing the digit $\varepsilon_{k}$ by $\varepsilon_{k}-1$. With each representation of $n$ such that $\varepsilon_{k}=0$, we associate a representation of $n-(\varepsilon+1) G_{k-1}$, replacing $\varepsilon_{k-1}$ by $\varepsilon_{k-1}-(\varepsilon+1)$. Thence

$$
\begin{aligned}
f(n) & \leq f\left(n-G_{k}\right)+f\left(n-(\varepsilon+1) G_{k-1}\right) \\
& \leq C\left(n-G_{k}\right)+C\left(n-(\varepsilon+1) G_{k-1}\right),
\end{aligned}
$$

and we obtain $f(n) \leq C n$ from inequalities (3.1) and (3.2).

REMARK. The above proposition works, for instance, in the case of $\phi$ being a Pisot or Salem number, because in that case $\alpha>1 \geq \delta^{\prime}=\delta$. Besides, considering the example introduced in [Fr], namely, $G_{k}=3 G_{k-1}+2 G_{k-2}+$ $3 G_{k-4}$ with positive initial values and $q=4$, we make sure that $\alpha>\delta^{\prime}$, although $\phi$ is neither Pisot nor Salem number.

4. Lipschitz exponent for $H$. We assume the same hypotheses as in Section 3 to be satisfied, and also suppose that the constant $\alpha=\log _{\phi} q$ is greater than $\delta^{\prime}=\delta+\log _{\phi} \varrho$.

TheOREM 4.1. There exists a constant $C>0$ such that

$$
\left|H\left(t^{\prime}\right)-H(t)\right| \leq C\left|t^{\prime}-t\right|^{\alpha-\delta^{\prime}}|\log | t^{\prime}-t||^{\alpha^{\prime}}
$$

for $\left|t^{\prime}-t\right| \leq 1 / 2$.

We first prove

Lemma 4.2. For $x \geq 1$,

(i) $\Phi(x+1)-\Phi(x)=O\left(x^{\delta}\right)$,

(ii) $\Phi(x)=O\left(x^{\delta+1}\right)$.

Proof. The first inequality follows from the hypothesis on $f$, and the second one is deduced from the first with the help of the inequality

$$
\Phi(x)-\Phi(1) \leq \sum_{n=1}^{[x]}(\Phi(n+1)-\Phi(n)) .
$$


Proof (of Theorem 4.1). As $H$ is periodic, we can confine ourselves to $1 \leq t<2$ and $t<t^{\prime} \leq t+1 / 2$. We have $\phi^{t^{\prime}}-\phi^{t}<\phi^{3}$. Furthermore, there exists a nonnegative integer $h$ such that $\phi^{2} \leq\left(\phi^{t^{\prime}}-\phi^{t}\right) \phi^{h}<\phi^{3}$. We set $x=\phi^{t+h}$ and $x^{\prime}=\phi^{t^{\prime}+h}$; then $\phi \leq x<x^{\prime}<x^{2}$. From Theorem 3.1 we get

$$
\left|\Phi(x)-x^{\alpha} H\left(\log _{\phi} x\right)\right|=O\left(x^{\delta^{\prime}}(\log x)^{\alpha^{\prime}}\right)
$$

and the same inequality for $x^{\prime}$, whence

$$
\begin{aligned}
\left|H\left(t^{\prime}\right)-H(t)\right|= & \left|H\left(\log _{\phi} x^{\prime}\right)-H\left(\log _{\phi} x\right)\right| \\
\leq & \left(x^{\prime}\right)^{-\alpha}\left(\Phi\left(x^{\prime}\right)-\Phi(x)\right)+\left|\left(x^{\prime}\right)^{-\alpha}-x^{-\alpha}\right| \Phi(x) \\
& +O\left(x^{\delta^{\prime}-\alpha}\left(\log x^{\prime}\right)^{\alpha^{\prime}}\right) .
\end{aligned}
$$

We have $0<x^{\prime}-x<\phi^{3}$, whence we deduce from Lemma 4.2(i) that the first term is $O\left(\left(x^{\prime}\right)^{\delta-\alpha}\right)$; it is also $O\left(x^{\delta-\alpha}\right)$, as, by hypothesis, $\delta-\alpha<-\log _{\phi} \varrho \leq 0$. The second term, in view of the mean value theorem and Lemma 4.2(ii), is $O\left(x^{-\alpha-1} \Phi(x)\right)=O\left(x^{\delta-\alpha}\right)$. Finally, as $\delta \leq \delta^{\prime}$ and $1<x<x^{\prime}<x^{2}$,

$$
\left|H\left(t^{\prime}\right)-H(t)\right|=O\left(x^{\delta^{\prime}-\alpha}(\log x)^{\alpha^{\prime}}\right) .
$$

It remains to estimate $x=\phi^{t+h}$. By the mean value theorem, we have

$$
\phi^{t} \log \phi<\frac{\phi^{t^{\prime}}-\phi^{t}}{t^{\prime}-t}<\phi^{t^{\prime}} \log \phi<\phi^{t+1} \log \phi,
$$

whence, by the definition of $h$, there exist positive constants $C_{1}$ and $C_{2}$ such that

$$
\frac{C_{1}}{t^{\prime}-t}<x<\frac{C_{2}}{t^{\prime}-t}
$$

which completes the proof of Theorem 4.1.

COROLlary 4.3. If $\phi$ is a Pisot number, then $H$ belongs to the Lipschitz class with exponent $\alpha-\delta$, where $\alpha=\log _{\phi} q$ and $f(x)=O\left(x^{\delta}\right)$.

\section{Properties of $F_{Y}$ for some cases of $\phi$}

LEMMA 5.1. Let

$$
\widetilde{Y}\left(\varepsilon_{0}, \varepsilon_{1}, \ldots\right):=Y-\mathbf{E} Y=\sum_{i=0}^{\infty}\left(\varepsilon_{i}-\frac{q-1}{2}\right) \phi^{-i} .
$$

Then the characteristic function of $\tilde{Y}$ is given by the formula

$$
\mathcal{F}_{\widetilde{Y}}(t)=\prod_{k=0}^{\infty} \frac{\sin \left(q \phi^{-k} t / 2\right)}{q \sin \left(\phi^{-k} t / 2\right)} .
$$

Proof. $\widetilde{Y}$ being a sum of independent random variables, its characteristic function is the product of their characteristic functions, which we 
compute using the well-known expression for the Dirichlet kernel $D_{n}(x)=$ $\sum_{k=-n}^{n} e^{i k x}$ (see, e.g., $[\mathrm{Ru}]$ ).

Proposition 5.2. If $\phi$ is an irrational Pisot number, then $\mathcal{F}_{\widetilde{Y}}(t)$ does not tend to 0 as $t \rightarrow \infty$, and thus, $Y$ is singular.

Proof. Let $t_{N}=2 \pi \phi^{N}$. Following [E], we will show that $\mathcal{F}_{\widetilde{Y}}\left(t_{N}\right) \nrightarrow 0$ as $N \rightarrow \infty$. Let $\|x\|:=\min \{|x-n|: n \in \mathbb{Z}\}$; we have

$$
\begin{aligned}
\mathcal{F}_{\widetilde{Y}}\left(t_{N}\right) & =\prod_{k=0}^{\infty} \frac{\sin \left(\pi q \phi^{N-k}\right)}{q \sin \left(\pi \phi^{N-k}\right)}=\prod_{j=1}^{N} \frac{\sin \left(\pi q \phi^{j}\right)}{q \sin \left(\pi \phi^{j}\right)} \cdot \prod_{k=0}^{\infty} \frac{\sin \left(\pi q \phi^{-k}\right)}{q \sin \left(\pi \phi^{-k}\right)} \\
& = \pm \mathcal{F}_{\widetilde{Y}}(2 \pi) \cdot \prod_{j=1}^{N} \frac{\sin \left(\pi q\left\|\phi^{j}\right\|\right)}{q \sin \left(\pi\left\|\phi^{j}\right\|\right)}
\end{aligned}
$$

whence in view of the fact that $\phi$ is an irrational Pisot number, there exists an irrational $\theta \in(0,1)$ such that $\left\|\phi^{j}\right\| \leq \theta^{j}$ for $j \geq N_{0}$. Hence

$$
\left|\mathcal{F}_{\widetilde{Y}}\left(t_{N}\right)\right| \geq\left|\mathcal{F}_{\widetilde{Y}}(2 \pi)\right| \cdot\left|\prod_{j=N_{0}+1}^{\infty} \frac{\sin \left(\pi \theta^{j}\right)}{q \sin \left(\pi \theta^{j}\right)}\right| \cdot\left|\prod_{j=1}^{N_{0}} \frac{\sin \left(\pi q\left\|\phi^{j}\right\|\right)}{q \sin \left(\pi\left\|\phi^{j}\right\|\right)}\right|>0,
$$

as $\left|\mathcal{F}_{\widetilde{Y}}(2 \pi)\right|>0$ by the irrationality of $\phi$.

Now we are going to study the same problem for the case of integral $\phi=: d$. We assume that $G_{k}=d^{k}$.

Proposition 5.3. Let $d$ be an integer. Then the function $F_{Y}$ is absolutely continuous if and only if $d$ divides $q$.

Proof. Let, within this proof,

$$
\mathcal{G}_{\nu}(u):=\frac{\sin \nu u}{\nu \sin u} .
$$

Consider the following cases.

1. $q=d^{m}$. Here

$$
\mathcal{G}_{q}(u)=\prod_{j=0}^{m-1} \mathcal{G}_{d}\left(d^{j} u\right)
$$

hence,

$$
\mathcal{F}_{\widetilde{Y}}(t)=\prod_{k=0}^{\infty} \mathcal{G}_{q}\left(d^{-k} t / 2\right)=\prod_{j=0}^{m-1}\left(\prod_{k=0}^{\infty} \mathcal{G}_{d}\left(d^{-k} \cdot d^{j} t / 2\right)\right)
$$

i.e.

$$
\widetilde{Y}=U\left[-\frac{1}{2}, \frac{1}{2}\right] * U\left[-\frac{d}{2}, \frac{d}{2}\right] * \ldots * U\left[-\frac{d^{m-1}}{2}, \frac{d^{m-1}}{2}\right],
$$

where $U[a, b]$ denotes the uniform distribution on $[a, b]$. Therefore, the random variable $\widetilde{Y}$ is absolutely continuous, and $\operatorname{supp} \widetilde{Y}=\left[-\frac{q-1}{2(d-1)}, \frac{q-1}{2(d-1)}\right]$. 
2. Let now $q$ be not divisible by $d$. Here, as in Proposition 5.2, taking $t_{N}=2 \pi d^{N}$, we have

$$
\mathcal{F}_{\widetilde{Y}}\left(t_{N}\right)= \pm \prod_{j=1}^{\infty} \frac{\sin \left(\pi q d^{-j}\right)}{q \sin \left(\pi d^{-j}\right)},
$$

and the assertion follows from the fact that $\sin \left(\pi q d^{-j}\right) \neq 0$ for any $j \geq 1$ by the hypothesis on $q$ and $d$.

3. Finally, if $q=d^{m} p$ with $p \geq 2$ and $p$ not divisible by $d$, then

$$
\mathcal{G}_{q}\left(d^{-k} t / 2\right)=\mathcal{G}_{d^{m}}\left(d^{-k} t / 2\right) \cdot \mathcal{G}_{p}\left(d^{-k} \cdot d^{m} t / 2\right),
$$

and the statement for this case follows from items 1,2 , as $\tilde{Y}$ is the convolution of the absolutely continuous distribution and the singular one, and, thus, it is itself absolutely continuous.

As a consequence, we obtain the result making Theorem 2.1 more precise.

Proposition 5.4. If $\phi$ is an irrational Pisot number or equals a positive integer $d$ and in this latter case $G_{k}=d^{k}$, and $d$ does not divide $q$, then $F_{Y}$ is singular, and $H$ is not differentiable on a certain continuum of points.

The question of for which bases the function $H$ is differentiable everywhere seems to be difficult and remains open. The only sufficient condition that we know is $G_{k}=d^{k}$ and $d$ divides $q$ (see above).

ExAmple. Let $d=2, q=6$. Here $\widetilde{Y}$ is the convolution of the uniform distribution $U[-1 / 2,1 / 2]$ and the singular one for the case " $d=2, q=3$ " studied below in detail. Thus, the function $f$ for this case has no asymptotics; this can be shown directly by proving that $f\left(2^{k}\right) \sim \frac{1}{6} \cdot 3^{k}$, and $f\left(2^{k}+2^{k-1}\right) \sim$ $3^{k-1}$, which in turn implies that there is no limit of the ratio $f(n) / n^{\log _{2} 3}$ as $n \rightarrow \infty$, though this ratio is bounded.

\section{Case of a radix base}

6.1. General facts. Let $d \geq 2, q \geq 2$ be integers, and let, as above, for any integer $n$,

$$
f(n)=\#\left\{\varepsilon_{0} \varepsilon_{1} \ldots: 0 \leq \varepsilon_{i} \leq q-1, \sum_{i=0}^{\infty} \varepsilon_{i} d^{i}=n\right\} .
$$

We also define, for any $x \in \mathbb{R} \backslash\{0\}$,

$$
\varphi(x)=\sum_{n \geq 0} f(n) x^{n} .
$$

Expanding the infinite product $\prod_{k \geq 0}\left(1+x^{d^{k}}+x^{2 d^{k}}+\ldots+x^{(q-1) d^{k}}\right)$, we find that it is equal to $\varphi(x)$; hence

$$
\varphi(x)=\left(1+x+x^{2}+\ldots+x^{q-1}\right) \varphi\left(x^{d}\right) .
$$


Since $f(n)$ is zero for $n<0$, we obtain

$$
\sum_{n \in \mathbb{Z}} f(n) x^{n}=\sum_{n \in \mathbb{Z}} f(n)\left(x^{d n}+x^{d n+1}+\ldots+x^{d n+q-1}\right) .
$$

Let $\{x\}=x-[x]$ (the fractional part of $x$ ) for any $x \in \mathbb{R}$. So, it follows from formula (6.1) that

$$
f(d n+r)= \begin{cases}\sum_{i=0}^{[(q-1) / d]-1} f(n-i), & r>d\{(q-1) / d\}, \\ \sum_{i=0}^{[(q-1) / d]} f(n-i), & r \leq d\{(q-1) / d\},\end{cases}
$$

for every $n \in \mathbb{Z}$ and every $r=0,1, \ldots, d-1$. Furthermore, if $[(q-1) / d]=0$, the first case in (6.2) turns into $f(d n+r)=0$ for $r>q-1$. We deduce from (6.2) by induction

$$
f(n) \leq n^{\beta}, \quad \beta=\log _{d}\left[\frac{q-1+d}{d}\right],
$$

and by Theorem 3.1, the following corollary holds (as $\varrho=1$ and $\alpha^{\prime}=0$ ).

Proposition 6.1.

$$
\Phi(x)=x^{\alpha} H\left(\log _{d} x\right)+O\left(x^{\beta}\right)
$$

with $\alpha=\log _{d} q$ and $\beta=\log _{d}[(q-1+d) / d]$.

REMARKs. 1. We always have $\alpha-1 \leq \beta<\alpha$, with equality iff $d$ divides $q$. In the case $q \leq d$, we have $\beta=0$ and it is easy to see that the function $f$ is $d$-automatic in the sense of [Co]: $(f(n))_{n \geq 0}$ is a fixed point, beginning with the letter 1 , of the substitution

$$
\begin{aligned}
& 0 \rightarrow 0 \ldots 0 \quad(d \text { times } 0), \\
& 1 \rightarrow 1 \ldots 10 \ldots 0 \quad(q \text { times } 1, d-q \text { times } 0) .
\end{aligned}
$$

The a.e. differentiability of $H$ is a consequence of Theorem 2.1. Note that this substitution is not primitive, as for a primitive substitution it was proved in $[\mathrm{DuT}]$ that $H$ is nowhere differentiable.

Below we will suppose $q>d$.

2. In the general case, the sequence $(f(n))_{n \geq 0}$ is $d$-regular in the sense of Allouche and Shallit [AllS]. Indeed, by (6.1) we are in the situation of Becker's Theorem 2 (see [Be]). The authors wish to express their thanks to J. P. Allouche for indicating the last-mentioned paper.

6.2. Relation between the order of $f$ and the order of the remainder term. In this subsection we are going to show that in the radix case it suffices to present the best possible upper bound of the order of $f$ for obtaining the best possible order of the remainder term. 
We first rewrite formula (6.2) by means of some transition matrices $M_{r}$, $r=0,1, \ldots, d-1$. Let $M_{r}$ be the $a \times a$ matrix, where $a$ is the minimal integer such that $a \geq(q-1) /(d-1)$. For $i=1, \ldots, a$, let

$$
\lambda_{r, i}=\frac{i+d-r-2}{d}, \quad \lambda_{r, i}^{\prime}= \begin{cases}\frac{q-1}{d}, & \left\{\lambda_{r, i}\right\}+\left\{\frac{q-1}{d}\right\}<1-\frac{1}{d}, \\ \frac{q-1}{d}+1, & \text { otherwise. }\end{cases}
$$

Then the $i$ th row of $M_{r}$ is defined as

$$
\left(M_{r}\right)_{i, j}= \begin{cases}1, & {\left[\lambda_{r, i}\right]+1 \leq j \leq\left[\lambda_{r, i}\right]+\left[\lambda_{r, i}^{\prime}\right]} \\ 0, & \text { otherwise }\end{cases}
$$

This definition makes sense if the integer $\left[\lambda_{r, i}\right]+\left[\lambda_{r, i}^{\prime}\right]$ is at most $a$ for any $i$; or, equivalently, if it is less than $a+1$. Now we deduce from the definition of $\lambda_{r, i}^{\prime}$ that $\left[\lambda_{r, i}\right]+\left[\lambda_{r, i}^{\prime}\right] \leq(a+d-2) / d+q / d$. The inequality $(a+d-2) / d+q / d<$ $a+1$ is valid, because it is equivalent to $a>(q-2) /(d-1)$.

We denote by $\mathbf{v}(n)$ the vector $\left(\begin{array}{c}f(n) \\ \vdots \\ f(n-a+1)\end{array}\right)$, for any $n \geq 0$.

Lemma 6.2. Any integer $n=\varepsilon_{k} d^{k}+\ldots+\varepsilon_{0}$ with $0 \leq \varepsilon_{i} \leq d-1$ satisfies

$$
\mathbf{v}(n)=M_{\varepsilon_{0}} \ldots M_{\varepsilon_{k}}\left(\begin{array}{c}
1 \\
0 \\
\vdots \\
0
\end{array}\right) \text {. }
$$

Proof. It is sufficient to show that $\mathbf{v}(n)=M_{\varepsilon_{0}} \mathbf{v}\left(n^{\prime}\right)$ with $n^{\prime}$ such that $n=d n^{\prime}+\varepsilon_{0}$. Let $i \in\{1, \ldots, a\}$ and let $i^{\prime}$, $i^{\prime \prime}$ be such that $i+d-\varepsilon_{0}-2=$ $d i^{\prime}+i^{\prime \prime}, 0 \leq i^{\prime \prime} \leq d-1$. We have $n+1-i=d\left(n^{\prime}-i^{\prime}\right)+\left(d-1-i^{\prime \prime}\right)$ whence by $(6.2)$,

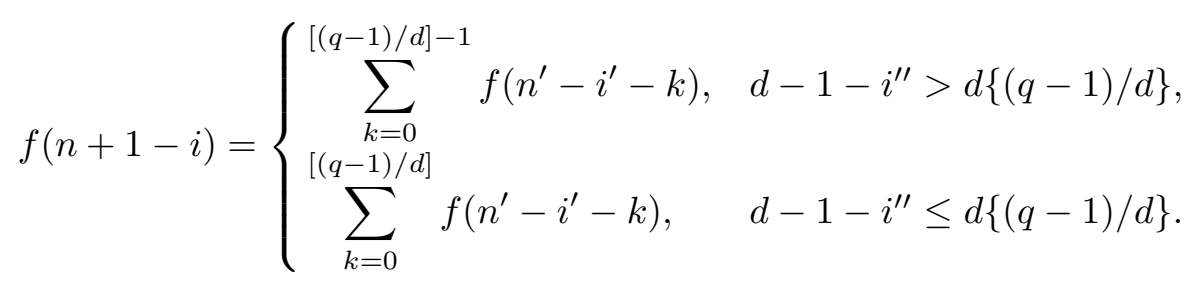

This coincides with the product of the $i$ th row of $M_{\varepsilon_{0}}$ by $\mathbf{v}\left(n^{\prime}\right)$.

Lemma 6.3. The matrix $M=M_{0}+\ldots+M_{d-1}$ is primitive, and its Perron-Frobenius eigenvalue equals $q$.

Proof. Let

$$
M\left(\begin{array}{c}
1 \\
\vdots \\
1
\end{array}\right)=\left(\begin{array}{c}
x_{1} \\
\vdots \\
x_{d}
\end{array}\right)
$$


We have

$$
x_{j}=d\left[\frac{q-1}{d}\right]+\#\left\{i: 0 \leq i \leq d-1,\left\{\lambda_{i, j}\right\}+\left\{\frac{q-1}{d}\right\} \geq \frac{d-1}{d}\right\} .
$$

Since $i \mapsto\left\{\lambda_{i, j}\right\}$ is a bijection from $\{0,1, \ldots, d-1\}$ to $\left\{\frac{0}{d}, \frac{1}{d}, \ldots, \frac{d-1}{d}\right\}$, we have

$$
x_{j}=d\left[\frac{q-1}{d}\right]+d\left\{\frac{q-1}{d}\right\}+1=q .
$$

We see that $q$ is an eigenvalue of $M$, and the other eigenvalues have moduli at most $q$.

For $M$ to be primitive, it suffices that the entries $M_{i, i-1}, M_{i, i}$ and $M_{i, i+1}$ are positive for any $i$ (because this implies $\left(M^{a}\right)_{i, j}>0$ for any $i$ and $j$ ). We consider the $i$ th row of $M$. We have $\left[\lambda_{r, i}^{\prime}\right] \geq 1$ for any $r$ and $i$; hence $\left(M_{r}\right)_{i,\left[\lambda_{r, i}\right]+1}=1$. The set $J_{i}=\left\{j: M_{i, j}>0\right\}$ is the union, for $r=$ $0, \ldots, d-1$, of $\left\{j:\left(M_{r}\right)_{i, j}>0\right\}$, the latter being an interval in $\mathbb{N}$ containing $\left[\lambda_{r, i}\right]+1$. As $\left[\lambda_{r+1, i}\right]-\left[\lambda_{r, i}\right]$ is 0 or $-1, J_{i}$ is also an interval.

It is sufficient to check that $\left[\lambda_{d-1, i}\right]+1<i<\left[\lambda_{0, i}\right]+\left[\lambda_{0, i}^{\prime}\right]$ for $i=1, \ldots, a$, except $\left[\lambda_{d-1,1}\right]+1=1$ and $\left[\lambda_{0, a}\right]+\left[\lambda_{0, a}^{\prime}\right]=a$. The first inequality is equivalent to $\lambda_{d-1, i}<i-1$, thence to $i>1$. For the second, we first deduce from the definition of $\left[\lambda_{0, i}\right]$ that $\left[\lambda_{0, i}\right]+\left[\lambda_{0, i}^{\prime}\right]$ is at least $\lambda_{0, i}+$ $(q-1) / d-(1-2 / d)$, thence it is $>i$ iff $i<(q-1) /(d-1)$, i.e. iff $i<a$. We obtain easily the two last-mentioned relations.

Proposition 6.4. Let $R(x)$ be the remainder term in Proposition 6.1. Then

$$
\inf \left\{\delta: R(x)=O\left(x^{\delta}\right)\right\}=\inf \left\{\delta: f(n)=O\left(n^{\delta}\right)\right\} .
$$

Proof. We first compute $H\left(\log _{d} x\right)$ for integral $x$. By (2.1), it is

$$
\lim _{k \rightarrow \infty} \frac{\Phi\left(x d^{k}\right)}{x^{\log _{d} q} q^{k}}
$$

Let $n$ be an integer such that $0 \leq n<x d^{k}$; we have $n=m d^{k}+r$ with $0 \leq m<x, 0 \leq r<d^{k}$. Let $r=\sum_{i=0}^{k-1} \varepsilon_{i} d^{i}$ be the canonical representation of $r$ in base $d$. From Lemma 6.2 we get

$$
\mathbf{v}(n)=M_{\varepsilon_{0}} \ldots M_{\varepsilon_{k-1}} \mathbf{v}(m) .
$$

Summing for $0 \leq r<d^{k}, 0 \leq m<x$, we obtain

$$
\left(\begin{array}{c}
\Phi\left(x d^{k}\right) \\
\vdots \\
\Phi\left(x d^{k}-a+1\right)
\end{array}\right)=M^{k}\left(\begin{array}{c}
\Phi(x) \\
\vdots \\
\Phi(x-a+1)
\end{array}\right) .
$$

By Lemma 6.3, $q^{-k} M^{k}$ tends to a matrix with positive entries; let $\alpha_{1} \ldots \alpha_{a}$ 
be its first row, and $\beta_{i}=\sum_{j=i}^{a} \alpha_{j}$ for any $i$. Then

$$
\begin{aligned}
H\left(\log _{d} x\right) & =x^{-\alpha}\left(\alpha_{1} \Phi(x)+\ldots+\alpha_{a} \Phi(x-a+1)\right) \\
& =x^{-\alpha}\left(\beta_{1} \Phi(x)-\beta_{2} f(x-1)-\ldots-\beta_{a} f(x-a+1)\right) .
\end{aligned}
$$

We have

$$
\begin{aligned}
R(x) & =\Phi(x)-x^{\alpha} H\left(\log _{d} x\right) \\
& =\left(1-\beta_{1}\right) \Phi(x)+\beta_{2} f(x-1)+\ldots+\beta_{a} f(x-a+1) .
\end{aligned}
$$

Now $1-\beta_{1}$ is zero, otherwise $R(x)$ would have the same order as $\Phi(x)$. The hypothesis $q>d$ implies $a \geq 2$, whence

$$
f(x-1) \ll R(x) \ll f(x-1)+\ldots+f(x-a+1) .
$$

This proves Proposition 6.4 for $x$ integer.

It is also true for any $x$ real: let $n$ be the minimal integer greater than or equal to $x$; we have $R(n)-R(x)=n^{\alpha} H\left(\log _{d} n\right)-x^{\alpha} H\left(\log _{d} x\right)$ and, using Theorem 4.1, we conclude that it is $O\left(x^{\delta}\right)$ for any $\delta$ such that $f(n)=$ $O\left(n^{\delta}\right)$.

REMARK. Some upper and lower bounds for $f(n)$ depending on the transition matrices under consideration are given in [D, p. 160, 161, 165].

6.3. Lipschitz exponent for $H$. Now we will prove that the same situation takes place also for the Lipschitz exponent of the function $H$. Let $\delta_{f}=\inf \{\delta$ : $\left.f(n)=O\left(n^{\delta}\right)\right\}$, and $\gamma_{H}=\sup \left\{\gamma:\left|H\left(t^{\prime}\right)-H(t)\right|=O\left(\left|t^{\prime}-t\right|^{\gamma}\right)\right\}$.

Corollary 6.5. If $\delta_{f}>\alpha-1$, then $\gamma_{H}=\alpha-\delta_{f}$.

Proof. For any $\varepsilon>0$ we have, by Theorem 4.1,

$$
\left|H\left(t^{\prime}\right)-H(t)\right| \ll\left|t^{\prime}-t\right|^{\alpha-\delta_{f}-\varepsilon} .
$$

Let $x$ be an integer, $t^{\prime}=\log _{d}(x+1)$ and $t=\log _{d} x$; we deduce from (6.3) that

$$
(x+1)^{\alpha} H\left(t^{\prime}\right)-x^{\alpha} H(t) \geq \alpha_{1} f(x), \quad(x+1)^{\alpha} H\left(t^{\prime}\right) \geq \alpha_{1} \Phi(x+1),
$$

and

$$
\begin{aligned}
H\left(t^{\prime}\right)-H(t)= & \left((x+1)^{-\alpha}-x^{-\alpha}\right)(x+1)^{\alpha} H\left(t^{\prime}\right) \\
& +x^{-\alpha}\left((x+1)^{\alpha} H\left(t^{\prime}\right)-x^{\alpha} H(t)\right) \\
\geq & -\alpha x^{-\alpha-1} \alpha_{1} \Phi(x+1)+x^{-\alpha} \alpha_{1} f(x) .
\end{aligned}
$$

As $\Phi(x) \asymp x^{\alpha}$ and $f(x) \geq x^{\delta_{f}-\varepsilon}$ for infinitely many $x$, we deduce

$$
\left|H\left(t^{\prime}\right)-H(t)\right| \gg x^{\delta_{f}-\alpha-\varepsilon} \gg\left|t^{\prime}-t\right|^{\alpha-\delta_{f}+\varepsilon},
$$

whence $\alpha-\delta_{f}-\varepsilon \leq \gamma_{H} \leq \alpha-\delta_{f}+\varepsilon$.

REMARK. This corollary cannot be applied in the case when $d$ divides $q$ : by Proposition 6.8 (see below) we have $\delta_{f}=\alpha-1$. Moreover, if $q=d^{m}$, then by Proposition 6.7 below we also have $H(t)=$ constant and $\gamma_{H}=\infty$. 
6.4. The case of $d$ dividing q; reduction. In this subsection we give an arithmetic version of Proposition 5.3. Let us first study the radix case with $q=d^{m}$ in more detail. Namely, we will give a precise asymptotic formula for $f$ in this case. Let $f_{m}^{(d)}(n)$ denote the number of representations of $n$ in base $d$ with digits $0,1, \ldots, d^{m}-1$. Whenever it is clear what $d$ is considered, we write simply $f_{m}(n)$.

LEMMA 6.6. If $m \geq 2, n \geq 0$ and $d \geq 2$, then

$$
f_{m}(n)=\sum_{j=0}^{[n / d]} f_{m-1}(j) .
$$

Proof. Fix $n \in \mathbb{N}$. Let $\varepsilon_{0} \varepsilon_{1} \varepsilon_{2} \ldots$ be a representation of $n$ in base $d$ with digits $0,1, \ldots, d^{m}-1$. We consider an integer $j \leq[n / d]$ and a sequence $\varepsilon_{0}^{\prime} \varepsilon_{1}^{\prime} \varepsilon_{2}^{\prime} \ldots$ which is the representation of $j$ in base $d$ with digits $0,1, \ldots, d^{m-1}-1$ defined as $\varepsilon_{i}^{\prime}:=\left[\varepsilon_{i} / d\right]$. The correspondence $\varepsilon_{0} \varepsilon_{1} \varepsilon_{2} \ldots \mapsto$ $\left(j, \varepsilon_{0}^{\prime} \varepsilon_{1}^{\prime} \varepsilon_{2}^{\prime} \ldots\right)$ is one-to-one, because the $\varepsilon_{i}^{\prime \prime}:=\varepsilon_{i}-d \varepsilon_{i}^{\prime}$ are just the digits of the canonical representation of $n-d j$ in base $d$.

Proposition 6.7. If $q=d^{m}$, then

$$
f_{m}(n)=\frac{d^{-m(m-1) / 2}}{(m-1) !} n^{m-1}+O\left(n^{m-2}\right), \quad n \rightarrow \infty,
$$

and

$$
H(t) \equiv \frac{d^{-m(m-1) / 2}}{m !}=\frac{q^{-(m-1) / 2}}{m !} .
$$

Proof. Note first that $f_{2}(n)=n / d+O(1)$ (a straightforward consequence of Lemma 6.6). Moreover, by induction and the above lemma, we have, for $m \geq 3$,

$$
\begin{aligned}
f_{m}(n) & =\sum_{j=0}^{[n / d]}\left[\frac{d^{-(m-1)(m-2) / 2}}{(m-2) !} j^{m-2}+O\left(j^{m-3}\right)\right] \\
& =\frac{d^{-(m-1)(m-2) / 2}}{(m-1) !}\left(\frac{n}{d}\right)^{m-1}+O\left(n^{m-2}\right) .
\end{aligned}
$$

This clearly implies the first part of the proposition. We deduce the second one from the relation

$$
\Phi(x)=\sum_{n<x} f_{m}(n)=\frac{d^{-m(m-1) / 2}}{m !} x^{m}+O\left(x^{m-1}\right)
$$

and (2.1).

Let $f_{u}^{*}(n)$ denote the number of representations of a nonnegative integer $n$ in fixed base $d$ with digits $0,1, \ldots, u-1$. 
Proposition 6.8. The following relation holds:

$$
f_{d u}^{*}(n)=\sum_{j=0}^{[n / d]} f_{u}^{*}(j) .
$$

The exact order of $f_{d u}^{*}(n)$ is $n^{\delta}$ with $\delta=\log _{d} u$.

Pr o of. We obtain this relation in the same way as in Lemma 6.6. Thence the function $f_{d u}^{*}(n)$ itself is the summation function $\Phi_{u}^{*}$, and Theorem 2.1 yields its precise asymptotic order.

EXAMPLES. 1. For any $d, f_{2}^{(d)}(d n)=n+1, f_{3}^{(d)}\left(d^{2} n\right)=(d n+2)(n+1) / 2$, etc.

2. If $d=2$, then $f_{3}(4 n)=(n+1)^{2}, f_{3}(4 n+2)=(n+1)(n+2) ; f_{4}(8 n)=$ $\sum_{k=0}^{n}(2 k+1)^{2}=(n+1)(2 n+1)(2 n+3) / 3, f_{4}(8 n+2)=f_{4}(8 n)+(n+1)^{2}$, $f_{4}(8 n+4)=f_{4}(8 n+2)+(n+1)(n+2), f_{4}(8 n+6)=f_{4}(8 n+4)+(n+1)(n+2)$, $f_{4}(8 n+8)=f_{4}(8 n+6)+(n+2)^{2}$, etc.

Conclusion. Thus, we can reduce a radix case with $d$ dividing $q$ to the case when $q>d$ and $q$ not divisible by $d$. In turn, for the latter case it suffices to find the best possible estimate for $f$ in order to solve the summation problem completely. In the present paper we will study some basic examples and plan to return to this problem in subsequent work.

For $d$ dividing $q$, as shown in Proposition 6.8, the maximal order of $f$ is exactly $O\left(n^{\beta}\right)$. It is curious that such a situation can arise even if $d$ does not divide $q$.

6.5. The precise maximal order of $f$ in the case $q \leq d^{2}$. We associate with any integer $n$ the integer $n^{\prime}=[n / d]$. Let $r:=[(q-1) / d]$, and $s:=$ $d\{(q-1) / d\}$.

Proposition 6.9. Let $q \leq d^{2}$. We have two cases.

(1) $f(n)=O\left(n^{\log _{d}(r+1)}\right)$ for $r \leq s$.

(2) $f(n)=O\left(n^{\log _{d} \frac{r+\sqrt{r^{2}+4 s+4}}{2}}\right)$ for $r>s$.

Both maximal orders are precise.

Pro of. We consider both cases simultaneously. From (6.2) we have

$$
f(n)= \begin{cases}\sum_{i=0}^{r-1} f\left(n^{\prime}-i\right), & n \in\{s+1, \ldots, d-1\} \bmod d, \\ \sum_{i=0}^{r} f\left(n^{\prime}-i\right), & n \in\{0, \ldots, s\} \bmod d .\end{cases}
$$

Let $I_{k}$ be the interval $\left\{d^{k}-d, \ldots, d^{k+1}-1\right\}, k \geq 0$. We denote by $X_{k}$ the maximum of $f(n)$ for $n \in I_{k}$ and $n \in\{0, \ldots, s\} \bmod d$, by $Y_{k}$ its maximum 
for $n \in I_{k}$ and $n \in\{s+1, \ldots, d-1\} \bmod d$. The hypothesis $q \leq d^{2}$ implies $r \leq d-1$ and hence, given $n \in I_{k}$, the integers $n^{\prime}-i$ belong to $I_{k-1}$ for any $i \in\{0, \ldots, r\}$, and at most $s+1$ of them belong to $\{0, \ldots, s\} \bmod d$ (or at most $r+1$ if $r \leq s$ ). For any $k \geq 1$, we obtain

$$
\begin{aligned}
X_{k} & \leq(s+1) X_{k-1}+(r-s) Y_{k-1} \quad \text { if } r \geq s+1, \\
Y_{k} & \leq(s+1) X_{k-1}+(r-s-1) Y_{k-1} \quad \text { if } r \geq s+1, \\
X_{k} & \leq(r+1) X_{k-1} \quad \text { if } r \leq s .
\end{aligned}
$$

Now we suppose $r \geq s+1$ (hence $r \geq 1$ and $s \leq d-2$ ). We consider the integers

$$
\begin{gathered}
n_{k}=\left\{\begin{array}{c}
s d+r d^{2}+\ldots+s d^{k-1}+r d^{k}, \quad k \text { even, } \\
r d+s d^{2}+\ldots+s d^{k-1}+r d^{k}, \quad k \text { odd, }
\end{array}\right. \\
m_{k}= \begin{cases}n_{k}+r, & k \text { even, } \\
n_{k}-1, & k \text { odd },\end{cases}
\end{gathered}
$$

with $n_{0}=0$. They satisfy the relations

$$
\begin{aligned}
f\left(n_{k}\right) & =(s+1) f\left(n_{k-1}\right)+(r-s) f\left(m_{k-1}\right), \\
f\left(m_{k}\right) & =(s+1) f\left(n_{k-1}\right)+(r-s-1) f\left(m_{k-1}\right),
\end{aligned}
$$

whence we obtain by induction $X_{k} \leq f\left(n_{k}\right)$ and $Y_{k} \leq f\left(m_{k}\right)$ for any $k \geq 0$. Actually, we have equality (as $n_{k}$ belongs to $I_{k}$ and to $\{0, \ldots, s\} \bmod d$, we cannot have $\left.f\left(n_{k}\right)>X_{k}\right)$.

From the first relation we obtain the expression for $f\left(m_{k-1}\right)$ in terms of $f\left(n_{k}\right)$ and $f\left(n_{k-1}\right)$ and, bringing it into the second relation, we deduce that for $k \geq 1$,

$$
f\left(n_{k+1}\right)=r f\left(n_{k}\right)+(s+1) f\left(n_{k-1}\right)
$$

with $f\left(n_{0}\right)=1$ and $f\left(n_{1}\right)=r+1$, hence

$$
f\left(n_{k}\right) \asymp\left(\frac{r+\sqrt{r^{2}+4 s+4}}{2}\right)^{k} .
$$

As $k=\log _{d} n+O(1)$ for $n \in I_{k}$, we see that

$$
f(n)=O\left(n^{\log _{d} \frac{r+\sqrt{r^{2}+4 s+4}}{2}}\right)
$$

is the precise maximal order of $f(n)$.

For $r \leq s$, we obtain in the same way

$$
f(n)=O\left(n^{\log _{d}(r+1)}\right) .
$$

REMARK. It is possible to show that the estimate $f(n)=O\left(n^{\beta}\right)$ can be improved for any $d$ and $q$ such that $d\{(q-1) / d\}<[(q-1) / d]$. We restrict ourselves to mentioning a better estimate for the case $d=2$ and arbitrary 
odd $q \geq 3$. In the same way as before it may be shown that

$$
X_{k} \leq\left[\frac{q+3}{4}\right] X_{k-1}+\left(\left[\frac{q+1}{4}\right] \cdot \frac{q-1}{2}\right) X_{k-2},
$$

whence $f(n)=O\left(n^{\widetilde{\beta}}\right)$, where

$$
\widetilde{\beta}=\log _{2} \frac{\left[\frac{q+3}{4}\right]+\sqrt{\left[\frac{q+3}{4}\right]^{2}+2(q-1)\left[\frac{q+1}{4}\right]}}{2}<\beta .
$$

Note that for $q=5$ this estimate yields $f(n)=O\left(n^{\log _{2}(\sqrt{3}+1)}\right)=O\left(n^{1.450}\right)$, while below we will show that the best possible estimate is approximately $O\left(n^{1.344}\right)$ (see Proposition 6.16).

As a corollary of the previous proposition, we obtain the corresponding result for some natural case.

Corollary 6.10. For $d=2, q=3$ the precise maximal order of $f$ is

$$
f(n)=O\left(n^{\log _{2} \frac{\sqrt{5}+1}{2}}\right) .
$$

Below we depict the representation graph for this case (see Introduction). It proves to be the Cayley graph for the semigroup $\langle a, b, c \mid a c=b a, b c=c a\rangle$.

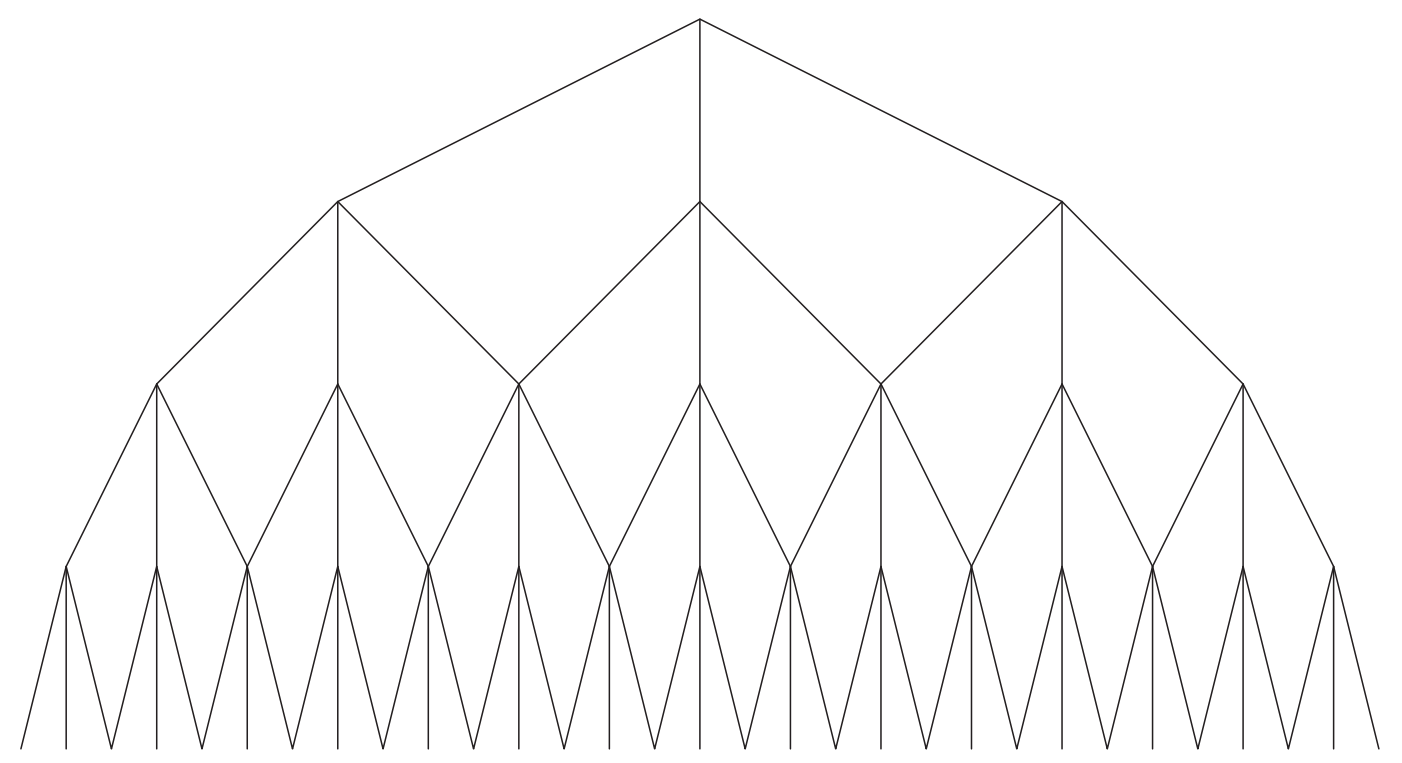

Fig. 1. The representation graph for $d=2, q=3$

In a sense this graph contains all finite continued fractions. Let us establish the precise link between the number of representations in the case $d=2, q=3$ and the regular continued fractions. 
Proposition 6.11. Let $n=\sum_{j=0}^{k} \varepsilon_{j} 2^{j}$ be the ordinary binary expansion of $n$ with $\varepsilon_{k}=1, \varepsilon_{j} \in\{0,1\}$. We rewrite it, grouping 0 's and 1's as

$$
\left(\varepsilon_{0}, \ldots, \varepsilon_{k}\right)=\left(1^{a_{0}} 0^{a_{1}} 1^{a_{2}} \ldots 0^{a_{s-1}} 1^{a_{s}}\right)
$$

with $a_{0} \geq 0\left(a_{0}=0\right.$ means that $n$ is even $)$, and $a_{i} \geq 1,1 \leq i \leq s$. We construct the regular continued fraction

$$
\frac{1}{a_{1}+\frac{1}{a_{2}+\ldots+\frac{1}{a_{s}}}}=: \frac{p_{s}}{q_{s}} .
$$

Then for $d=2, q=3$ we have $f(n)=q_{s}$.

Pr o of. From formula (6.2) applied to the case in question, we have

$$
\left(\begin{array}{c}
f(n) \\
f(n-1)
\end{array}\right)=\left(\begin{array}{ll}
1 & 1 \\
0 & 1
\end{array}\right)\left(\begin{array}{c}
f\left(n^{\prime}\right) \\
f\left(n^{\prime}-1\right)
\end{array}\right)
$$

if $n$ is even, and

$$
\left(\begin{array}{c}
f(n) \\
f(n-1)
\end{array}\right)=\left(\begin{array}{ll}
1 & 0 \\
1 & 1
\end{array}\right)\left(\begin{array}{c}
f\left(n^{\prime}\right) \\
f\left(n^{\prime}-1\right)
\end{array}\right)
$$

otherwise. Therefore,

$$
\begin{aligned}
& \left(\begin{array}{c}
f(n) \\
f(n-1)
\end{array}\right) \\
& \quad=\left(\begin{array}{cc}
1 & 0 \\
a_{0} & 1
\end{array}\right)\left(\begin{array}{cc}
1 & a_{1} \\
0 & 1
\end{array}\right) \ldots\left(\begin{array}{cc}
1 & a_{s-1} \\
0 & 1
\end{array}\right)\left(\begin{array}{cc}
1 & 0 \\
a_{s} & 1
\end{array}\right)\left(\begin{array}{l}
1 \\
0
\end{array}\right) \\
& \quad=\left(\begin{array}{ll}
0 & 1 \\
1 & 0
\end{array}\right)\left(\begin{array}{cc}
a_{0} & 1 \\
1 & 0
\end{array}\right)\left(\begin{array}{cc}
a_{1} & 1 \\
1 & 0
\end{array}\right) \ldots\left(\begin{array}{cc}
a_{s-1} & 1 \\
1 & 0
\end{array}\right)\left(\begin{array}{cc}
a_{s} & 1 \\
1 & 0
\end{array}\right)\left(\begin{array}{l}
1 \\
0
\end{array}\right) .
\end{aligned}
$$

The relation of such a matrix product to the continued fractions is wellknown (see, e.g., $[\mathrm{Po}]$ ).

6.6. The case $d=2, q=5$. In this case the situation is more complicated. We consider it in detail in order to show the sort of difficulties that may appear when processing a kind of recurrent sequences arising in such problems.

We define the maximal order of $f$ in this case as

$$
\delta_{f}=\inf \left\{\delta: f(n)=O\left(n^{\delta}\right)\right\} .
$$

We obtain easily $f(0)=f(1)=1, f(2)=f(3)=2, f(4)=4, f(5)=3$, $f(6)=5$ and $f(7)=4$. Let $n=\varepsilon_{k} 2^{k}+\ldots+\varepsilon_{0}$ with $\varepsilon_{i} \in\{0,1\}$ and $\varepsilon_{k} \neq 0$, and let $n^{\prime}=\varepsilon_{k} 2^{2}+\varepsilon_{k-1} 2^{1}+\varepsilon_{k-2} 2^{0} \in\{4,5,6,7\}$. From Lemma 6.2 we 
deduce that for $n \geq 8$,

$$
\left(\begin{array}{c}
f(n) \\
f(n-1) \\
f(n-2) \\
f(n-3)
\end{array}\right)=M_{\varepsilon_{0}} \ldots M_{\varepsilon_{k-3}}\left(\begin{array}{c}
f\left(n^{\prime}\right) \\
f\left(n^{\prime}-1\right) \\
f\left(n^{\prime}-2\right) \\
f\left(n^{\prime}-3\right)
\end{array}\right)
$$

with

$$
M_{0}=\left(\begin{array}{llll}
1 & 1 & 1 & 0 \\
0 & 1 & 1 & 0 \\
0 & 1 & 1 & 1 \\
0 & 0 & 1 & 1
\end{array}\right) \quad \text { and } \quad M_{1}=\left(\begin{array}{cccc}
1 & 1 & 0 & 0 \\
1 & 1 & 1 & 0 \\
0 & 1 & 1 & 0 \\
0 & 1 & 1 & 1
\end{array}\right)
$$

Let the norm of a matrix $M=\left(m_{i, j}\right)_{1 \leq i, j \leq 4}$ be defined as

$$
\|M\|=\max _{1 \leq j \leq 4} \sum_{i=1}^{4}\left|m_{i, j}\right|
$$

and the corresponding vector norm $\|\mathbf{v}\|:=\sum_{i=1}^{4}\left|v_{i}\right|$. Let

$$
\delta_{h}:=\max \left\{\frac{1}{h+1} \log _{2}\left(\left\|M_{\varepsilon_{0}} \ldots M_{\varepsilon_{h}}\right\|\right):\left(\varepsilon_{0}, \ldots, \varepsilon_{h}\right) \in\{0,1\}^{h+1}\right\} .
$$

From (6.4) and the values of $f(0), f(1), \ldots, f(7)$ we deduce that

$$
\begin{aligned}
\left\|M_{\varepsilon_{0}} \ldots M_{\varepsilon_{k-3}}\right\| & \leq f(n)+f(n-1)+f(n-2)+f(n-3) \\
& \leq 16\left\|M_{\varepsilon_{0}} \ldots M_{\varepsilon_{k-3}}\right\|,
\end{aligned}
$$

whence $\delta_{f}=\lim \sup _{h \rightarrow \infty} \delta_{h}$.

Let $M_{0}^{\prime}$ and $M_{1}^{\prime}$ denote the transposes of $M_{0}$ and $M_{1}$ respectively. Since $c_{1}\|M\| \leq\left\|M^{\prime}\right\| \leq c_{2}\|M\|$ for any matrix $M$ with some absolute constants $c_{1}, c_{2}$, we have

(6.5) $\delta_{f}=\limsup _{k \rightarrow \infty}\left\{\frac{1}{k} \log _{2} \max \left(\left\|M_{\varepsilon_{k}}^{\prime} \ldots M_{\varepsilon_{0}}^{\prime} \mathbf{v}\right\|\right):\left(\varepsilon_{0}, \ldots, \varepsilon_{k}\right) \in\{0,1\}^{k+1}\right\}$

with an arbitrary vector $\mathbf{v} \in \mathbb{N}^{4}$. We now compute this limit.

Let

$$
\varphi_{\mathbf{v}}\left(\varepsilon_{0}, \ldots, \varepsilon_{k}\right):=\left\|M_{\varepsilon_{k}}^{\prime} M_{\varepsilon_{k-1}}^{\prime} \ldots M_{\varepsilon_{0}}^{\prime} \mathbf{v}\right\|
$$

for any $\left(\varepsilon_{0}, \ldots, \varepsilon_{k}\right) \in\{0,1\}^{k+1}$. Let, by definition, $V_{4}=\left\{\left(v_{1}, v_{2}, v_{3}, v_{4}\right) \in\right.$ $\left.\mathbb{N}^{4}: v_{1}+v_{4} \leq \min \left(v_{2}, v_{3}\right)\right\}$. Note that $M_{0}^{\prime} \mathbf{v} \in V_{4}$ and $M_{1}^{\prime} \mathbf{v} \in V_{4}$ for any $\mathbf{v} \in V_{4}$.

Proposition 6.12. Let $\mathbf{v} \in V_{4}$. Then for $k \geq 2$,

$$
\max _{\varepsilon_{1}, \ldots, \varepsilon_{k} \in\{0,1\}^{k}} \varphi_{\mathbf{v}}\left(0, \varepsilon_{1}, \ldots, \varepsilon_{k}\right)= \begin{cases}\varphi_{\mathbf{v}}(010 \ldots 101), & k \text { odd }, \\ \varphi_{\mathbf{v}}(010 \ldots 010), & k \text { even }\end{cases}
$$


and

$$
\max _{\varepsilon_{1}, \ldots, \varepsilon_{k} \in\{0,1\}^{k}} \varphi_{\mathbf{v}}\left(1, \varepsilon_{1}, \ldots, \varepsilon_{k}\right)= \begin{cases}\varphi_{\mathbf{v}}(1010 \ldots 10), & k \text { odd }, \\ \varphi_{\mathbf{v}}(1010 \ldots 01), & k \text { even } .\end{cases}
$$

We first prove three lemmas.

LEMMA 6.13.

$$
\begin{aligned}
\varphi_{\mathbf{v}}\left(\varepsilon_{0}, \ldots, \varepsilon_{k}\right)=a v_{1}+b v_{2}+c v_{3}+d v_{4} \\
\quad \Rightarrow \varphi_{\mathbf{v}}\left(1-\varepsilon_{0}, \ldots, 1-\varepsilon_{k}\right)=d v_{1}+c v_{2}+b v_{3}+a v_{4} .
\end{aligned}
$$

Proof. This is an application of the fact that the matrices $M_{0}^{\prime}$ and $M_{1}^{\prime}$ are "centrally symmetric", i.e. $\left(M_{0}^{\prime}\right)_{i, j}=\left(M_{1}^{\prime}\right)_{5-i, 5-j}$.

Thus, it suffices to prove the first claim of the above proposition, because the condition defining $V_{4}$ is invariant with respect to the simultaneous substitution $v_{1} \mapsto v_{4}, v_{2} \mapsto v_{3}$.

LEMMA 6.14.

$$
\varphi_{\mathbf{v}}(00) \leq \varphi_{\mathbf{v}}(01) \quad \text { and } \quad \varphi_{\mathbf{v}}(11) \leq \varphi_{\mathbf{v}}(10)
$$

for any $\mathbf{v} \in V_{4}$.

Proof. We have $\varphi_{\mathbf{v}}(00)=8 v_{1}+5 v_{2}+7 v_{3}+5 v_{4}$ and $\varphi_{\mathbf{v}}(01)=7 v_{1}+$ $5 v_{2}+8 v_{3}+5 v_{4}$, hence, $\varphi_{\mathbf{v}}(01)-\varphi_{\mathbf{v}}(00)=v_{3}-v_{1} \geq 0$. The second claim is a consequence of Lemma 6.13.

LEMMA 6.15.

$$
\varphi_{\mathbf{v}}\left(0, \varepsilon_{1}, \varepsilon_{2}\right) \leq \varphi_{\mathbf{v}}(010) \quad \text { and } \quad \varphi_{\mathbf{v}}\left(1, \varepsilon_{1}, \varepsilon_{2}\right) \leq \varphi_{\mathbf{v}}(101)
$$

for any $\varepsilon_{1}, \varepsilon_{2} \in\{0,1\}$ and any $\mathbf{v} \in V_{4}$.

Proof. Again, by Lemma 6.13, we may deal with $\varepsilon_{0}=0$. We have $\varphi_{\mathbf{v}}(000)=20 v_{1}+12 v_{2}+17 v_{3}+12 v_{4}, \varphi_{\mathbf{v}}(001)=20 v_{1}+13 v_{2}+18 v_{3}+13 v_{4}$, $\varphi_{\mathbf{v}}(010)=18 v_{1}+13 v_{2}+20 v_{3}+12 v_{4}$, and finally, $\varphi_{\mathbf{v}}(011)=17 v_{1}+12 v_{2}+$ $20 v_{3}+13 v_{4}$. Thus, since $\mathbf{v} \in V_{4}$, we have $\varphi_{\mathbf{v}}(010)-\varphi_{\mathbf{v}}(000)=v_{2}+3 v_{3}-$ $2 v_{1} \geq 0, \varphi_{\mathbf{v}}(010)-\varphi_{\mathbf{v}}(001)=2 v_{3}-2 v_{1}-v_{4} \geq 2\left(v_{3}-v_{1}-v_{4}\right) \geq 0$, and $\varphi_{\mathbf{v}}(010)-\varphi_{\mathbf{v}}(011)=v_{1}+v_{2}-v_{4} \geq 0$.

Proof of Proposition 6.12. We use induction on $k$. For $k=1,2$ it is just the consequence of the above two lemmas. Let $k \geq 3$ and suppose for simplicity it is odd. We have $\varphi_{\mathbf{v}}\left(\varepsilon_{0}, \ldots, \varepsilon_{k}\right)=\varphi_{\mathbf{v}^{\prime}}\left(\varepsilon_{1}, \ldots, \varepsilon_{k}\right)$, where $\mathbf{v}^{\prime}:=M_{\varepsilon_{0}}^{\prime} \mathbf{v} \in V_{4}$. Let, say, $\varepsilon_{0}=0$. We have two cases.

1. $\varepsilon_{1}=1$. By the induction hypothesis,

$$
\varphi_{\mathbf{v}^{\prime}}\left(1, \ldots, \varepsilon_{k}\right) \leq \varphi_{\mathbf{v}^{\prime}}(101 \ldots 01)=\varphi_{\mathbf{v}}(01 \ldots 01) .
$$

2. $\varepsilon_{1}=0$. Similarly,

$$
\varphi_{\mathbf{v}^{\prime}}\left(0, \ldots, \varepsilon_{k}\right) \leq \varphi_{\mathbf{v}}(\underbrace{00101 \ldots 010}_{k+1 \text { digits }}) .
$$


We are left to show that $\varphi_{\mathbf{v}}(00101 \ldots 010) \leq \varphi_{\mathbf{v}}(0101 \ldots 01)$. Let

$$
\begin{aligned}
\left.\varphi_{\mathbf{v}}(\underbrace{01 \ldots 01}_{k+1 \text { digits }}) \text { or } \varphi_{\mathbf{v}}(\underbrace{01 \ldots 010}_{k+1 \text { digits }}) \text { if } k \text { is even }\right) \\
=: \alpha_{k} v_{1}+\beta_{k} v_{2}+\gamma_{k} v_{3}+\delta_{k} v_{4} .
\end{aligned}
$$

Then, by Lemma 6.13,

$$
\varphi_{\mathbf{v}}(\underbrace{1010 \ldots 10}_{k+1 \text { digits }})=\delta_{k} v_{1}+\gamma_{k} v_{2}+\beta_{k} v_{3}+\alpha_{k} v_{4},
$$

hence, $\alpha_{0}=\beta_{0}=\gamma_{0}=\delta_{0}=1$, and

$$
\begin{aligned}
\alpha_{k+1} & =\beta_{k}+\gamma_{k}+\delta_{k}, & \gamma_{k+1} & =\alpha_{k}+\beta_{k}+\gamma_{k}, \\
\beta_{k+1} & =\beta_{k}+\gamma_{k}, & & \delta_{k+1}=\alpha_{k}+\beta_{k} .
\end{aligned}
$$

We have $\alpha_{k+4}=2 \alpha_{k+3}+2 \alpha_{k+2}-2 \alpha_{k+1}+\alpha_{k}$, and the same recurrence relation holds for $\beta_{k}, \gamma_{k}$ and $\delta_{k}$. Note that if a sequence satisfies this relation, and begins with four nondecreasing positive integers, then all of its terms are positive.

Moreover,

$$
\begin{aligned}
\varphi_{\mathbf{v}}(00101 \ldots 010)= & \varphi_{\mathbf{v}^{\prime}}(\underbrace{01 \ldots 010}_{k \text { digits }}) \\
= & \alpha_{k-1} v_{1}+\beta_{k-1}\left(v_{1}+v_{2}+v_{3}\right) \\
& +\gamma_{k-1}\left(v_{1}+v_{2}+v_{3}+v_{4}\right)+\delta_{k-1}\left(v_{3}+v_{4}\right) \\
= & \gamma_{k} v_{1}+\beta_{k} v_{2}+\alpha_{k} v_{3}+\left(\gamma_{k-1}+\delta_{k-1}\right) v_{4}
\end{aligned}
$$

(by (6.6)), whence

$$
\begin{aligned}
\varphi_{\mathbf{v}}(\underbrace{0101 \ldots 01}_{k+1 \text { digits }}) & -\varphi_{\mathbf{v}}(\underbrace{00101 \ldots 010}_{k+1 \text { digits }}) \\
= & \left(\gamma_{k}-\alpha_{k}\right)\left(v_{3}-v_{1}\right)-\left(\gamma_{k-1}+\delta_{k-1}-\alpha_{k-1}-\beta_{k-1}\right) v_{4} \\
& \geq\left(\gamma_{k}-\alpha_{k}\right)\left(v_{3}-v_{1}-v_{4}\right),
\end{aligned}
$$

as $\gamma_{k} \geq \alpha_{k}+1, k \geq 3$ (induction), and $\gamma_{k-1}+\delta_{k-1}-\alpha_{k-1}-\beta_{k-1} \leq$ $\gamma_{k}-\alpha_{k}=\alpha_{k-1}-\delta_{k-1}$ by (6.6) and by the above remark on positivity. Indeed, let $\varkappa_{k}:=2\left(\alpha_{k}-\delta_{k}\right)+\beta_{k}-\gamma_{k}$. Then $\varkappa_{1}=\varkappa_{2}=1, \varkappa_{3}=5, \varkappa_{4}=10$, $\varkappa_{5}=29$, etc. The proof is complete.

From this proposition and formula (6.5) it follows by Gelfand's celebrated formula $\varrho(M)=\lim _{n \rightarrow \infty}\left(\left\|M^{n}\right\|\right)^{1 / n}$ for the spectral radius $\varrho$ that

$$
\delta_{f}=\frac{1}{2} \log _{2} \varrho\left(M_{0} M_{1}\right)=\log _{2} \varrho(M)=1.344042 \ldots,
$$

where $M$ is taken from (6.6), i.e. $M=\left(\begin{array}{llll}0 & 1 & 1 & 1 \\ 0 & 1 & 1 & 0 \\ 1 & 1 & 1 & 0 \\ 1 & 1 & 0 & 0\end{array}\right)$. Note that $M^{2}=M_{0} M_{1}$. So, we proved the following result. 
Proposition 6.16. For $d=2, q=5$ the precise maximal order of the function $f$ is $f(n)=O\left(n^{\log _{2} \psi}\right) \approx n^{1.344}$, where $\psi$ is the unique root of the equation $x^{4}=2 x^{3}+2 x^{2}-2 x+1$ lying in the interval $(2,3)$.

6.7. Fibonacci case. To end the paper we consider an important integer case with a nonradix base, namely, the Fibonacci case.

Here $f(n)$ is the number of representations of $n$ as a sum of distinct Fibonacci numbers. This function was under consideration in [Ca], [AlZ] from the viewpoint of its combinatorial properties. Ergodic properties of the transformation of the interval called the "goldenshift", which is associated with the sequence $f(n)$, were studied in the recent work [SV].

The results for the Fibonacci case were mentioned at the beginning of the paper. They were obtained in the preprint $[\mathrm{S}]$; note that it contains a gap in the proof of the upper bounds. However, they are direct consequences of the estimate for $f(n)$ obtained in $[\mathrm{Pu}](f(n) \leq \sqrt{n+1})$ and Theorem 3.1 and Corollary 4.2 of the present paper, while the proof of the lower bounds for the Lipschitz exponent of $H$ and the order of the remainder term in [S] is correct.

Note that in this case $F$ is the distribution function of the well-known singular Erdős measure introduced in [E] and studied in detail in [AlZ] and recently in $[\mathrm{SV}]$.

As a consequence of the main result we easily obtain a new property of the Erdős measure.

Proposition 6.17. The Lipschitz exponent of the distribution function of the Erdös measure equals $\Lambda-1 / 2$.

Note that recently this result was obtained in $[\mathrm{LaNg}$, Theorem D] by using different techniques.

Open problems. We mention some related open problems.

Integer bases. 1. Find the best possible maximal order for $f(n)$ for an arbitrary radix case.

2. Associate natural transfer matrices with certain classes of nonradix bases, for instance, in the Pisot case.

3. Study the problem of the existence, for a fixed case like the case $d=2, q=3$, of an increasing sequence of integers with density 1 such that $\log f(n) / \log n$ tends to some constant as $n$ tends to infinity along this sequence. We claim that this problem can be solved by applying the multiplicative ergodic theorem of Furstenberg and Kesten to sequences of transfer matrices. Another problem that seems to be more difficult is to find the exact value of the constant in question. 
Noninteger bases. In $[\mathrm{KP}]$ a necessary and sufficient condition for the existence and uniqueness of representation in the ring $\mathbb{Z}[\alpha]$ with $\alpha$ being an algebraic integer over $\mathbb{Q}$ is given. It is worthwhile to consider the problem similar to the one studied in the present paper for the situation where the uniqueness condition is not satisfied, i.e. to deal with a certain greater alphabet lying in $\mathbb{Z}$.

\section{References}

[AlZ] J. C. Alexander and D. Zagier, The entropy of a certain infinitely convolved Bernoulli measure, J. London Math. Soc. 44 (1991), 121-134.

[AllS] J. P. Allouche and J. Shallit, The ring of k-regular sequences, Theoret. Comput. Sci. 98 (1992), 163-197.

[Be] P. G. Becker, k-regular power series and Mahler-type functional equations, J. Number Theory 49 (1994), 269-286.

[Bo] D. Bogdanov, Branching graph for the de Rham curve, Master Thesis, St. Petersburg State University, St. Petersburg, 1993.

[Br] N. G. de Bruijn, On Mahler's partition problem, Indag. Math. 10 (1948), $210-220$

[Bu] Y. Bugeaud, Sur la suite des nombres de la forme $\varepsilon_{0}+\varepsilon_{1} q+\ldots+\varepsilon_{n} q^{n}$, $\varepsilon_{i} \in\{0,1\}$, to appear.

[Ca] L. Carlitz, Fibonacci representations, Fibonacci Quart. 6 (1968), 193-220.

[Co] A. Cobham, Uniform tag sequences, Math. Systems Theory 6 (1972), 164-192.

[D] P. Dumas, Récurrences Mahlériennes, suites automatiques, et études asymptotiques, Doctorat de mathématiques, Université de Bordeaux, 1993.

[DF] P. Dumas et P. Flajolet, Asymptotique des récurrences mahlériennes: le cas cyclotomique, J. Théor. Nombres Bordeaux 8 (1996), 1-30.

[DuT] J. M. Dumont et A. Thomas, Systèmes de numération et fonctions fractales relatifs aux substitutions, Theoret. Comput. Sci. 65 (1989), 153-169.

[E] P. Erdős, On a family of symmetric Bernoulli convolutions, Amer. J. Math. 61 (1939), 974-976.

[Fr] C. Frougny, Representations of numbers and finite automata, Math. Systems Theory 25 (1992), 37-60.

[JW] B. Jessen and A. Wintner, Distribution functions and the Riemann zeta function, Trans. Amer. Math. Soc. 38 (1938), 48-88.

[KP] B. Kovács and A. Pethő, Number systems in integral domains, especially in orders of algebraic number fields, Acta Sci. Math. (Szeged) 55 (1991), 287-299.

$[\mathrm{LaNg}] \quad$ K.-S. Lau and S.-M. Ngai, $L^{q}$-spectrum of the Bernoulli convolution associated with the golden ratio, Studia Math. 131 (1998), 225-251.

[Po] A. J. van der Poorten, An introduction to continued fractions, in: Diophantine Analysis, London Math. Soc. Lecture Note Ser. 109, Cambridge Univ. Press, 1986, 99-138.

$[\mathrm{Pu}]$ I. Pushkarev, The ideal lattices of multizigzags and the enumeration of Fibonacci partitions, Zap. Nauchn. Sem. POMI 223 (1995), 280-312 (in Russian).

[Rh] G. de Rham, Sur une courbe plane, J. Math. Pures Appl. 35 (1956), 25-42.

[Ru] W. Rudin, Principles of Mathematical Analysis, McGraw-Hill, New York, 1964. 
[S] N. A. Sidorov, The summation function for the number of Fibonacci representations, PDMI preprint 15/1995, 1-17.

[SV] N. A. Sidorov and A. M. Vershik, Ergodic properties of the Erdös measure, the entropy of the goldenshift, and related problems, Monatsh. Math. 126 (1998), $215-261$.

Steklov Institute of Mathematics at St. Petersburg

27 Fontanka

St. Petersburg 191011, Russia

E-mail: sidorov@pdmi.ras.ru
Institut de Mathématiques de Luminy UPR 9016

Avenue de Luminy-Case 930 13288 Marseille Cedex 9, France E-mail: thomas@iml.univ-mrs.fr

Received on 2.12.1997

and in revised form on 20.6.1998 